\title{
Predictors of 15-Day Survival for the Intensive Care Unit Patient on Continuous Renal Replacement Therapy: A Retrospective Analysis
}

Abdul Hasan Siddiqui ${ }^{1}$, Gautam Valecha ${ }^{2}$, Jwalant Modi ${ }^{3}$, Amina Saqib ${ }^{4}$, Chanudi Weerasinghe ${ }^{5}$, Faraz Siddiqui ${ }^{6}$, Suzanne El Sayegh ${ }^{7}$

1. Pulmonary and Critical Care Medicine, University of Illinois Urbana Champaign, Champaign, USA 2. HematologyOncology, Staten Island University Hospital, Staten Island, USA 3. Nephrology, University of Cincinnati College of Medicine, Cincinnati, USA 4. Pulmonary/Critical Care, Robert Wood Johnson Hospital, New Brunswick, USA 5. Internal Medicine, Staten Island University Hospital, Staten Island, USA 6. Pulmonary and Critical Care Medicine, Robert Packer Hospital, Sayre, USA 7. Internal Medicine, Zucker School of Medicine at Hofstra Northwell, Staten Island University Hospital Northwell Health, Staten Island, USA

Corresponding author: Abdul Hasan Siddiqui, drsiddiqui07@gmail.com

\section{Abstract}

\section{Purpose}

In the intensive care unit (ICU), acute renal failure is mostly part of multiple organ dysfunction syndromes with mortality ranging from $28 \%-90 \%$, continuous renal replacement therapy (CRRT) is the predominant mode of RRT used in ICU. The main objective of the study was to evaluate the outcomes in patients with acute kidney injury (AKI) on CRRT in the ICU.

\section{Methods}

A retrospective chart review was conducted for all ICU patients with acute renal failure on CRRT in a tertiary care teaching hospital. A subgroup analysis was conducted between 15 days in hospital survivors and nonsurvivors to look for predictors of survival for patients on CRRT.

\section{Results}

Two-hundred twenty-six patients underwent CRRT from January 2007 to December 2013. The overall inhospital mortality was $84.1 \%$. Fifty-six patients (24.77\%) survived to the 15 -day post-CRRT mark. Acute respiratory failure requiring mechanical ventilation was associated with significantly increased mortality; $89.2 \%$ vs. $97.6 \%$ ( $\mathrm{P}=0.008$ ), ICU length of stay was significantly longer in the survivor group than the nonsurvivor group. Median $\pm \mathrm{IQR} ;\{20 \pm 24$ vs $6 \pm 7(\mathrm{P}:<0.0001)\}$ and so were the ventilator-associated days $\{16 \pm 24$ vs $4 \pm 6.5(\mathrm{P}:<0.0001)\}$ and duration of CRRT $\{4.5 \pm 5.5$ vs $2 \pm 2.0(\mathrm{P}:<0.0001)\}$. Patients who survived had a lower incidence of metabolic acidosis $\{44.6 \%$ vs $62.9 \%$ (P: 0.016$)\}$ and uremic encephalopathy $\{12.5 \%$ vs 26.5\%; (P: 0.031) $\}$ but a greater incidence of volume overload $\{28.6 \%$ vs $15.9 \%$ (P: 0.031$)\}$ as compared to the non-survivor. Acute Physiology And Chronic Health Evaluation II (APACHE II) scores were significantly higher in the non-survivor group (mean SD) $26.9 \pm 28.0$ vs. $23.9 \pm 25.8$ (P: 0.0136).

Received 05/04/2020

Review began 05/09/2020 Review ended 05/09/2020 Published 05/18/2020

๑) Copyright 2020

Siddiqui et al. This is an open access article distributed under the terms of the Creative Commons Attribution License CC-BY 4.0., which permits unrestricted use, distribution, and reproduction in any medium, provided the original author and source are credited.

\section{Conclusions}

This observational study in patients undergoing CRRT in an ICU setting revealed that the overall mortality was $84.1 \%$. Fluid overload as an indication of CRRT was associated with improved 15 days' survival whereas higher APACHE II scores and the use of mechanical ventilation were associated with reduced 15 days' survival.

Categories: Internal Medicine, Nephrology, Healthcare Technology

Keywords: continuous renal replacement therapy (crrt), cvvh, medical icu, mortality, mortality rate in sepsis, renal failure, aki, general nephrology dialysis and transplantation

\section{Introduction}

An acute kidney injury (AKI) requiring intermittent or continuous renal replacement therapy (RRT) significantly affects morbidity and mortality in critically ill patients and constitutes a substantial health care burden [1]. The mortality and morbidity associated with AKI remain to be a concern despite numerous improvements in RRT techniques and after significant advances in supportive intensive care unit (ICU) care. Importantly, the development of AKI constitutes an independent risk factor for death in the ICU.

Continuous RRT (CRRT) is widely used in ICUs and is often viewed as the superior approach in critically ill patients [1-4]. CRRTs refer to either dialysis (diffusion-based solute removal) or filtration (convection-based solute and water removal) treatments that operate continuously. The major advantage of continuous 
therapy is the slower rate of solute or fluid removal per unit of time. Thus, it has significant utility in hemodynamically unstable patients who cannot tolerate conventional intermittent hemodialysis [5]. This modality had theoretical advantages over intermittent renal replacement therapy, which are related to hemodynamic stability, optimal metabolic control, and fluid balance allowing nutritional supplementation. In ICU settings, AKI is mostly part of multiple organ dysfunction syndromes, and mortality in these patients ranges from $28 \%-90 \%[6-10]$.

Despite considerable advances in the management of critically ill patients and RRT, the high mortality rate in AKI could be explained, in part, by the individual characteristics of the patient population undergoing RRT. This may include a higher proportion of elderly patients, an increase in the severity and number of failing organs, and the delayed occurrence of AKI in patients whose survival is prolonged. However, despite the availability of CRRTs, the mortality associated with AKI in ICU patients on RRT remains high. So far, an insufficient number of studies have been conducted to identify the variable and characteristics that define the severity of illness and predict mortality in ICU patients on CRRT [11].

To better understand the characteristics of the patients undergoing CRRT, we conducted a study with the aim to establish an association between demographic characteristics and variables that define the severity of illness and the in-hospital and short-term mortality outcomes. We also identified a subgroup of patients that may benefit from CRRT regarding survival and quality of life post CRRT.

\section{Materials And Methods}

A single-center retrospective chart review was conducted on all adult patients above the age of 18 years undergoing CRRT at Staten Island University Hospital during the years 2007 to 2013.

Indications for initiating CRRT were fluid overload refractory to diuretics, hyperkalemia (serum potassium concentration $>6.5 \mathrm{mEq} / \mathrm{L}$ ), or rapidly rising potassium levels, refractory to medical therapy, severe metabolic acidosis $(\mathrm{pH}<7.1)$ in patients in whom the administration of bicarbonate was not indicated, such as those with volume overload, or those with lactic acidosis or ketoacidosis, in whom bicarbonate administration was ineffective and signs of uremia such as pericarditis, encephalopathy, or an otherwise unexplained decline in mental status. Drug Intoxication with hemodynamic instability was also used as indications for CRRT.

\section{Exclusion criteria}

Patients who could not tolerate CRRT for more than two hours were excluded from the study.

\section{Study oversight and confidentiality}

Each subject was assigned a unique consecutive number, not derived from any patient identifiers, known as the "code number." The code numbers were listed in a Master Subject Log and served to link the code number with their name, date of birth, and medical record number. RedCap (Vanderbilt University, Nashville, Tennessee) was used to enter all data. As it was a retrospective study with no intervention, a waiver of informed consent was submitted to the institutional review board (IRB). The study was subsequently approved by the IRB.

\section{Study design}

After including the eligible patients, their records were evaluated for various demographic and disease severity characteristics. The demographic characteristics assessed were age, sex, body mass index (BMI), and other medical co-morbidities, including coronary artery disease, heart failure, liver cirrhosis, hypertension, diabetes mellitus, end-stage renal disease (ESRD), and chronic obstructive pulmonary disease (COPD). Primary ICU diagnosis included the indication for ICU admission. Patients were also stratified based on acute respiratory failure requiring mechanical ventilation (noninvasive or invasive), number of pressors required, and the presence of sepsis. The length of ICU stay, indication for, and number of days on mechanical ventilation and CRRT were recorded for individual patients.

\section{Endpoints}

The primary endpoint included survival rate at 15 days after the termination of CRRT. Secondary endpoints included a three-month survival rate, new dialysis dependence post-discharge, worsening chronic kidney disease (CKD) from baseline, and length of ICU stay. APACHE II scores were calculated from the vital signs, oxygenation status, Glasgow coma scale (GCS), and laboratory parameters at the time of ICU admission. Lengths of ICU stay, indication for CRRT, and the number of days on mechanical ventilation and CRRT were recorded for individual patients.

\section{Statistical analysis}

Continuous variables were reported as the mean \pm standard deviation (SD) and categorical variables as frequency and percentages. A chi-square test was done for categorical variables. A descriptive analysis was 


\section{Cureus}

used to identify demographic data and clinical parameters. A subgroup analysis was performed between survivors and non-survivors to analyze the predictors of better outcomes with CRRT. All statistics were done using SAS software (SAS Institute, Cary, North Carolina). A P-value of $<0.05$ was considered significant and an independent predictor of better survival in patients on CRRT in the ICU.

\section{Results}

\section{Subject enrollment}

A total of 258 patients underwent CRRT at our institution from January 2007 to December 2013; 21 patients were excluded, as they didn't complete a minimum of two hours of CRRT. Two-hundred thirty-seven patients were included in the study, 11 patients had missing information related to demographics and outcomes, and hence only 226 patients were included in the final analysis.

\section{Baseline characteristics}

Among the 226 subjects n(\%), 127 (56.0) were males. The mean age of the population was mean \pm SD $66.8 \pm 14.2$. All 226 patients were in the Intensive care unit at the time of CRRT, and 43 (19) of them were postoperative surgical ICU patients. The majority of them had septic shock 164 (73) as their ICU diagnosis followed by acute respiratory failure150 (66) and gastrointestinal bleed 20 (9). See Table 1. 


\section{Cureus}

Baseline characteristics

Male sex $n(\%)$

127(56)

Female sex $\mathrm{n}(\%)$

99(44)

ICU diagnosis

$\mathrm{n}(\%)$

Acute respiratory failure

150(66)

Acute CHF

18(8)

Septic shock

164(73)

GI bleed/Hemorrhagic shock

20(9)

Arrhythmia

12(5)

ACS (STEMI/NSTEMI)

14(6)

Postop

43(19)

Intracranial bleed

Baseline comorbidities

Congestive heart failure

59(26)

End-stage renal disease

Diabetes mellitus

90(40)

Hypertension

156(69)

Cirrhosis

20(9)

Coronary artery disease

79(35)

COPD

Indications for CRRT

Refractory hyperkalemia

Metabolic acidosis $(\mathrm{ph}<7.2)$

132(58)

Uremic encephalopathy/Pericarditis

$53(23)$

Drug intoxication

$4(2)$

Refractory fluid overload

43(19)

\section{TABLE 1: Baseline characteristics of patients undergoing CRRT ( $N: 226)$}

N: sample size; $n$ : number of patients; STEMI: ST-segment elevation myocardial infarction; NSTEMI: non-ST-segment elevation myocardial infarction; ICU: intensive care unit; CHF: congestive heart failure; GI: gastrointestinal; ACS: acute coronary syndrome; COPD: chronic obstructive pulmonary disease; CRRT: continuous renal replacement therapy

Twenty-nine out of 226 (13\%) patients had ESRD at the time of presentation and were dialysis-dependent. Ninety-five percent of the patients required mechanical ventilation at the time of initiation of CRRT. The most common indication for RRT was severe metabolic acidosis 132 (58\%) followed by uremic encephalopathy 53 (23\%) and refractory fluid overload 43 (19\%).

\section{Primary outcome}

Overall in-hospital mortality was $84.1 \%$ (190/226). Only 56 patients (24.77\%) survived to the 15 -day postCRRT mark. Twenty out of 56 (35.71\%) patients died during the same hospitalization. Among the 36 who survived, 16 (28.57\%) were discharged home, 15 (26.7\%) were discharged to a skilled nursing facility, and five (8.9\%) were released to other destinations.

\section{Secondary outcomes and subgroup analysis}




\section{Cureus}

A subgroup analysis between the 15 days' survivor and non-survivor groups was conducted. Acute respiratory failure requiring mechanical ventilation was associated with significantly increased mortality; $89.2 \%$ vs. $97.6 \%$ ( $p=0.008$ ). Baseline comorbidities did not have a significant impact on 15 -day survival. The ICU length of stay was significantly longer in the survivor group than the nonsurvivor group median $\pm \mathrm{IQR}\{20 \pm 24$ vs. $6 \pm 7$ (P: $<0.0001)\}$ and so were the ventilator-associated days $\{16 \pm 24$ vs. $4 \pm 6.5$ (P: $<0.0001)\}$ and duration of CRRT $\{4.5 \pm 5.5$ vs. $2 \pm 2.0(\mathrm{P}:<0.0001)\}$. See Tables $2-3$.

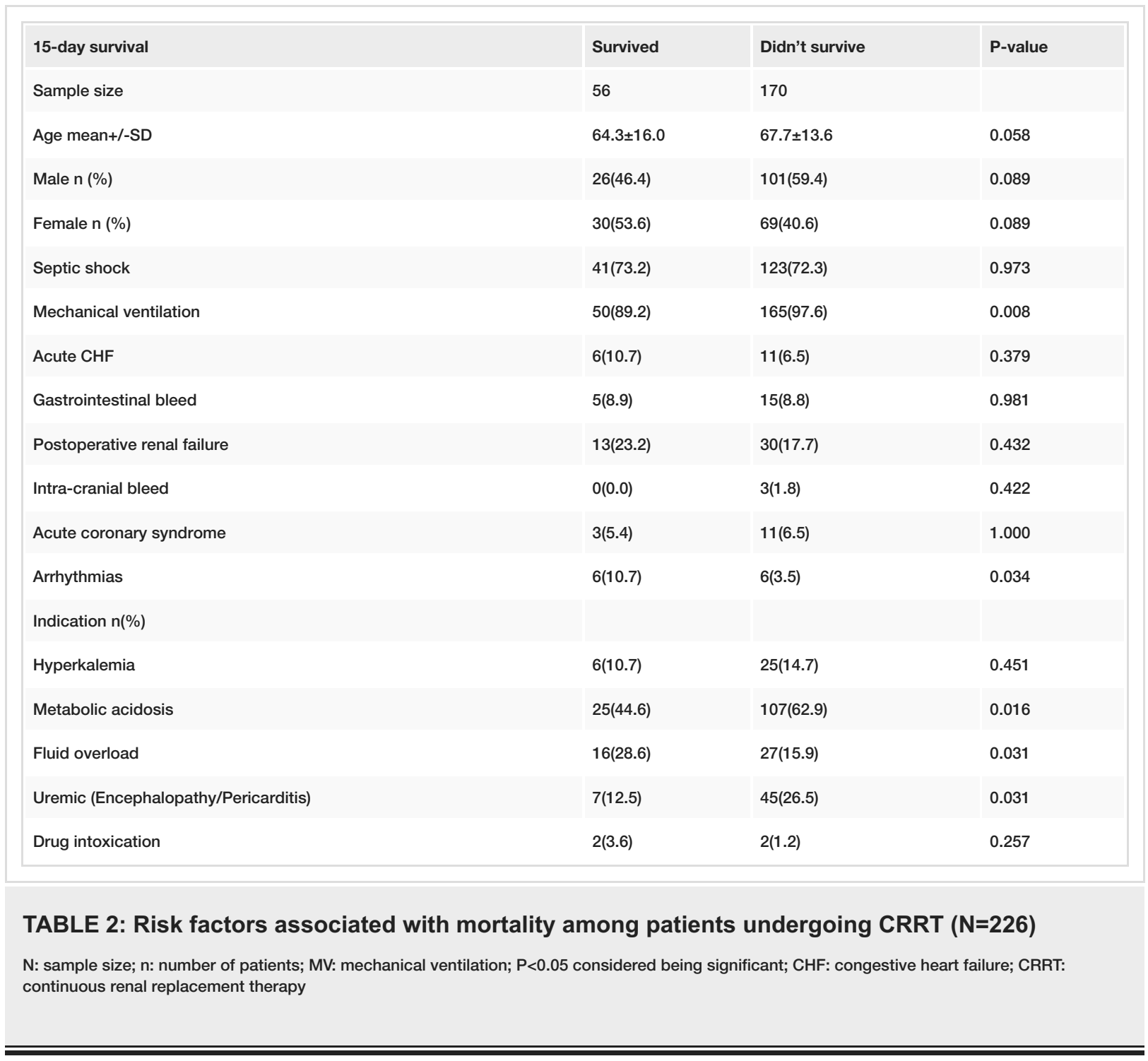




\section{Cureus}

\begin{tabular}{|c|c|c|c|}
\hline 15-day survival & Survived & Didn't survive & P-value \\
\hline Days on vent Median \pm IQR & $16 \pm 24$ & $4 \pm 6.5$ & $<0.0001$ \\
\hline CRRT duration (days) Median \pm IQR & $4.5 \pm 5.5$ & $2 \pm 2.0$ & $<0.0001$ \\
\hline ICU duration Median \pm IQR & $20 \pm 24$ & $6 \pm 7$ & $<0.0001$ \\
\hline Lactate Median \pm |QR & $2.8 \pm 4.1$ & $6 \pm 8.2$ & $<0.0001$ \\
\hline Platelets Median \pm IQR & $150 \pm 148$ & $120 \pm 134$ & 0.0374 \\
\hline Albumin Median \pm IQR & $1.9 \pm 0.8$ & $1.8 \pm 0.8$ & 0.0164 \\
\hline Creatinine Median \pm IQR & $3.7 \pm 2.5$ & $2.7 \pm 2.7$ & 0.5510 \\
\hline No of vasopressors $\geq 2: n(\%)$ & $36(64.3)$ & 137(81.5) & 0.037 \\
\hline APACHE II scores (Mean \pm SD) & $23.9 \pm 25.8$ & $26.9 \pm 28.0$ & 0.0136 \\
\hline
\end{tabular}

TABLE 3: Subgroup analysis of patients on CRRT between survivors and non-survivors

APACHE II: Acute Physiology And Chronic Health Evaluation II (acute physiology score + age points + chronic health points); CRRT: continuous renal replacement therapy

Diagnosis at the time of intensive care unit admission did not have a significant impact on 15-day survival among patients undergoing CRRT. Patients who survived had a lower incidence of metabolic acidosis $\{44.6 \%$ vs $62.9 \%$ (P: 0.016)\}, uremic encephalopathy $\{12.5 \%$ vs $26.5 \%$; (P: 0.031$)\}$ but a greater incidence of volume overload $\{28.6 \%$ vs $15.9 \%$ (P: 0.031$)\}$ as compared to the non-survivor. No significant difference was found for hyperkalemia $\{10.7 \%$ vs $14.7 \%$ (P: 0.451 ) between the two groups.

Patients who didn't survive for 15 days had elevated APACHE II scores, elevated lactate, low platelets, low albumin, and higher use of vasopressors as compared to those who survive. There was no significant difference between the creatinine levels of the two groups (Table 3).

\section{Discussion}

CRRT has become the choice of RRT for the treatment of AKI in patients admitted to ICUs. In this singlecenter retrospective review, the overall in-hospital mortality of patients undergoing CRRT in ICU was found to be $84.2 \%$, with only $24.8 \%$ of patients surviving the 15 -day post-CRRT mark. The reported mortality in patients undergoing CRRT in the ICU ranges from $37 \%$ to $75 \%$ [12-13]. Observed mortality in our study is higher than the reported death, probably due to the overall morbidity of our population observed by the fact that the majority of our patients (95.5\%) were on mechanical ventilation and $76.5 \%$ of patients were on more than or equal to two vasopressors at the time of initiation of CRRT. Moreover, these two factors were independently associated with increased mortality after 15 days. Furthermore, the majority of our patients were non-surgical ICU patients, with merely $19 \%$ of postoperative subjects in our study, and the sample size was too small to show any significant survival difference in this sub-group. A recent study published in JAMA in surgical ICU patients showed a mortality rate of $53.7 \%$ for patients undergoing CRRT in ICU [14].

Unlike other studies, the patients who survived the first 15 days received a longer duration of CRRT as compared to those who did not survive [14]. This may just reflect that patients who showed signs of recovery while being on CRRT were continued for longer durations before switching to regular hemodialysis.

Outcomes were unaffected by age and gender while some previous studies have associated advancing age and male sex with adverse outcomes. Acute respiratory failure requiring mechanical ventilation was associated with significantly increased mortality. As expected, high baseline APACHE II scores, low albumin, and elevated lactate levels were associated with increased mortality, which also reiterates findings from multiple previous studies [15-16]. In another study, the Liano score has been reported to identify a group of patients on dialysis for acute renal failure with a near $100 \%$ chance of mortality but did not show discrimination between those patients who died in hospital and those who did not [8,17]: Douma and associates showed in their report that the use of both the APACHE and Liano scores would be better at predicting hospital mortality [8].

Interestingly, baseline comorbidities did not have a significant impact on the 15-day mortality rate. Septic shock was the most common ICU diagnosis, and hyperkalemia was the most common indication for CRRT. Severe metabolic acidosis with $\mathrm{Ph}<7.2$ was associated with significantly increased mortality as compared to other indications for CRRT, whereas fluid overload as an indication for CRRT had better 15-day survival in 
our study. This could be explained by the fact that CRRT is best known for its fluid balance ability and patients with fluid overload as an indication for dialysis may have better outcomes as compared to other indications for dialysis.

In conjunction with other studies, our study also aims at determining the variables that may predict the short-term mortality rates in critically ill patients on CRRT. This, in turn, would help in identifying the patient population that would most likely benefit from this expensive treatment modality that may otherwise be a futile exercise in other patient populations, thereby controlling healthcare expenditure. However, predictors may vary with different subjects, depending upon the underlying condition. For example, in patients after cardiopulmonary bypass, lactate levels may rise regardless of the patient's condition or the presence of peripheral circulation insufficiency. In these patients, the cardiac index or presence of a low output syndrome will be a better predictor than the lactate levels.

It should also be noted that our retrospective chart review might not be used to determine a cause-effect relationship. Furthermore, large-scale multicenter studies with larger sample sizes are needed to evaluate the predictors better to set standards while employing CRRT.

\section{Conclusions}

In conclusion, the requirement of CRRT in ICU patients may portend a poor prognosis, as it may carry a very high risk of mortality of up to $84.1 \%$, as shown in our study population. Fluid overload as an indication of CRRT appears to be a better predictor of survival in patients undergoing CRRT. Patients with significant medical illness indicated by higher APACHE II scores may have worse outcomes and, therefore, the appropriate selection of patients for this procedure is of paramount importance, not only to improve survival but also for the efficient utilization of health care resources. Besides, this is a single-center observational study and, perhaps, the results cannot be extrapolated for the general population of the United States. However, more robust, multicenter randomized controlled clinical trials are needed to further evaluate the role of CRRT in patients with acute renal failure in the ICU.

\section{Additional Information \\ Disclosures}

Human subjects: Consent was obtained by all participants in this study. Animal subjects: All authors have confirmed that this study did not involve animal subjects or tissue. Conflicts of interest: In compliance with the ICMJE uniform disclosure form, all authors declare the following: Payment/services info: All authors have declared that no financial support was received from any organization for the submitted work. Financial relationships: All authors have declared that they have no financial relationships at present or within the previous three years with any organizations that might have an interest in the submitted work. Other relationships: All authors have declared that there are no other relationships or activities that could appear to have influenced the submitted work.

\section{References}

1. Bellomo R, Ronco C, Mehta RL, et al.: Acute kidney injury in the ICU: from injury to recovery: reports from the 5th Paris International Conference. Ann Intensive Care. 2017, 7:49. 10.1186/s13613-017-0260-y

2. Druml W: Acute renal failure is not a "cute" renal failure! . Intensive Care Med. 2004, 30:1886-1190. 10.1007/s00134-004-2344-z

3. Pannu N, Klarenbach S, Wiebe N, Manns B, Tonelli M: Renal replacement therapy in patients with acute renal failure. A systematic review. JAMA. 2008, 299:793-805. 10.1001/jama.299.7.793

4. Ricci Z, Ronco C: Timing, dose and mode of dialysis in acute kidney injury . Curr Opin Crit Care. 2011, 17:556-561. 10.1097/MCC.0b013e32834cd360

5. Bouchard J, Soroko SB, Chertow GM, et al.: Fluid accumulation, survival and recovery of kidney function in critically ill patients with acute kidney injury. Kidney Int. 2009, 76:422-427. 10.1038/ki.2009.159

6. Chuasuwan A, Gojaseni P, Chittinandana A: Continuous veno-venous hemofiltration in Bhumibol Adulyadej Hospital. J Med Assoc Thai. 2006, 89:86-97.

7. Wong DT, Knaus WA: Predicting outcome in critical care: the current status of the APACHE prognostic scoring system. Can J Anaesth. 1991, 38:374-383. 10.1007/BF03007629

8. Douma CE, Redekop WK, van der Meulen JH, et al.: Predicting mortality in intensive care patients with acute renal failure treated with dialysis. J Am Soc Nephrol. 1997, 8:111-7.

9. Chertow GM, Levy EM, Hammermeister KE, Grover F, Daley J: Independent association between acute renal failure and mortality following cardiac surgery. Am J Med. 1998, 104:343-348. 10.1016/s00029343(98)00058-8

10. Metnitz PG, Krenn CG, Steltzer H, Lang T Ploder J Lenz K, Le Gall J-R, Druml W: Effect of acute renal failure requiring renal replacement therapy on outcome in critically ill patients. Crit Care Med. 2002, 30:2051-2058. 10.1097/00003246-200209000-00016

11. Rabindranath K, Adams J, Macleod AM, Muirhead N: Intermittent versus continuous renal replacement therapy for acute renal failure in adults. Cochrane Database Syst Rev. 2007, 2007:CD003773. 10.1002/14651858.CD003773.pub3

12. Prasad B, Urbanski M, Ferguson TW, Karreman E, Tangri N: Early mortality on continuous renal replacement therapy (CRRT): the prairie CRRT study. Can J Kidney Health Dis. 2016, 3:36. 10.1186/s40697016-0124-7 


\section{Cureus}

13. Stads S, Fortrie G, van Bommel J, Zietse R, Betjes MG: Impaired kidney function at hospital discharge and long-term renal and overall survival in patients who received CRRT. Clin J Am Soc Nephrol. 2013, 8:12841291. 10.2215/CJN.06650712

14. Tatum JM, Barmparas G, Ko A, Dhillon N, Smith E, Margulies DR, Ley EJ: Analysis of survival after initiation of continuous renal replacement therapy in a surgical intensive care unit. JAMA Surg. 2017, 152:938-943. 10.1001/jamasurg.2017.1673

15. Sasaki S, Gando S, Kobayashi S, et al.: Predictors of mortality in patients treated with continuous hemodiafiltration for acute renal failure in an intensive care setting. Asaio J. 2001, 47:86-91. 10.1097/00002480-200101000-00018

16. Rajdev K, Leifer L, Sandhu G, et al.: Fluid resuscitation in patients with end-stage renal disease on hemodialysis presenting with severe sepsis or septic shock: a case control study. J Crit Care. 2020, 55:157162. 10.1016/j.jcrc.2019.10.008

17. Varricatt VP, Rau NR, Attur RP, Baig WW: Validation of Liano score in acute renal failure: a prospective study in Indian patients. Clin Exp Nephrol. 2009, 13:33-37. 10.1007/s10157-008-0073-2 